infinite slope with $\frac{1}{\gamma} \frac{\mathrm{d} c}{\mathrm{~d} z}=0.29$ and $c_{0}=0$. When $S=2 c_{0}$, the crest of the limiting slope is vertical downwards and for $S=(2+2 \pi) c_{0}$, the crest of the slope is vertical upwards. These two values of $S$ limit the range for which this analysis yields practicable solutions to this type of problem.

This plasticity analysis was developed to analyse the results obtained from model clay slopes tested on a large centrifuge. Data recorded during the test were interpreted to obtain the distribution of mobilized maximum shear stress within the plane section of the model. By using this distribution of shear stress as the function $c(x, z)$ the analysis could be used to calculate the complete stress distribution within the model at the given stage of the test. The profile of the slope was specified and solutions were obtained for the traction on the sloping face and the stresses within the soil at given stages of a test. The contours of shear stress at one stage of a test are shown in Fig. 2 and the solution obtained for the stresses is plotted in Fig. 3. At this stage of the test, the model, having been submerged in water, was being subjected to rapid drawdown. In Fig. 4 the water pressure on the slope face is plotted against depth and the stresses normal to the face of the slope as obtained from the solution are plotted. The agreement between the calculated and the actual water pressures is within the expected experimental accuracy.

\title{
REFERENCES
}

Endicott, L. J. (1970). Centrifugal testing of soil models. Ph.D. thesis, University of Cambridge.

\section{Force portante d'un sol sur une assise rigide (étude théorique)}

MANDEL, J. and SALENÇON, J. (1972). Géotechnique 22, No. 1, 79-93.

\section{J. R. Booker, Department of Civil Engineering, University of Sydney}

The Authors have found a numerical solution for the case in which the average horizontal stress corresponds to passive failure in regions remote from the footing. The Writer has obtained an analytic solution (Booker, 1972) to a different case in which it is assumed that the average horizontal stress is zero. The different cases are of course related. Let $P_{\text {av }}^{\prime}$ denote

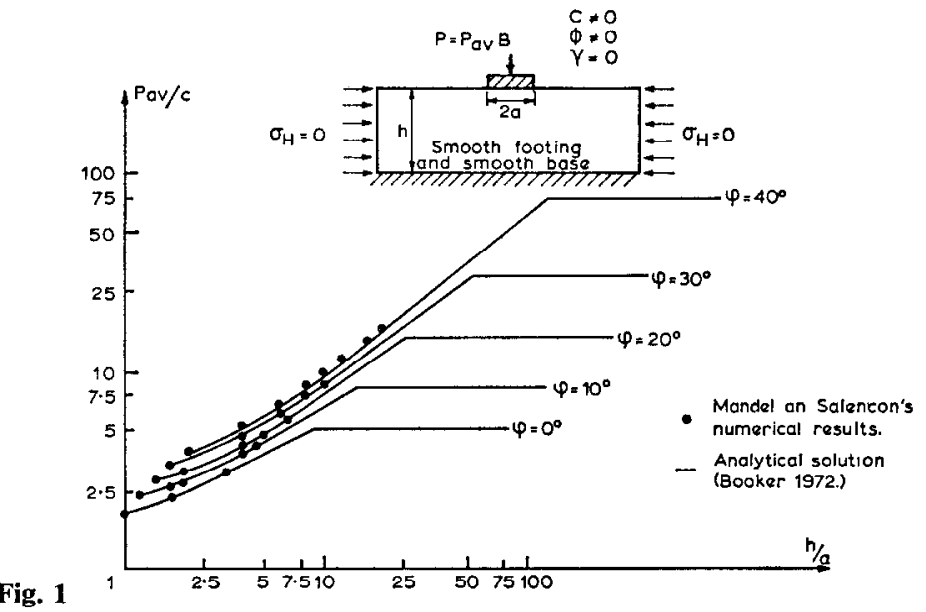




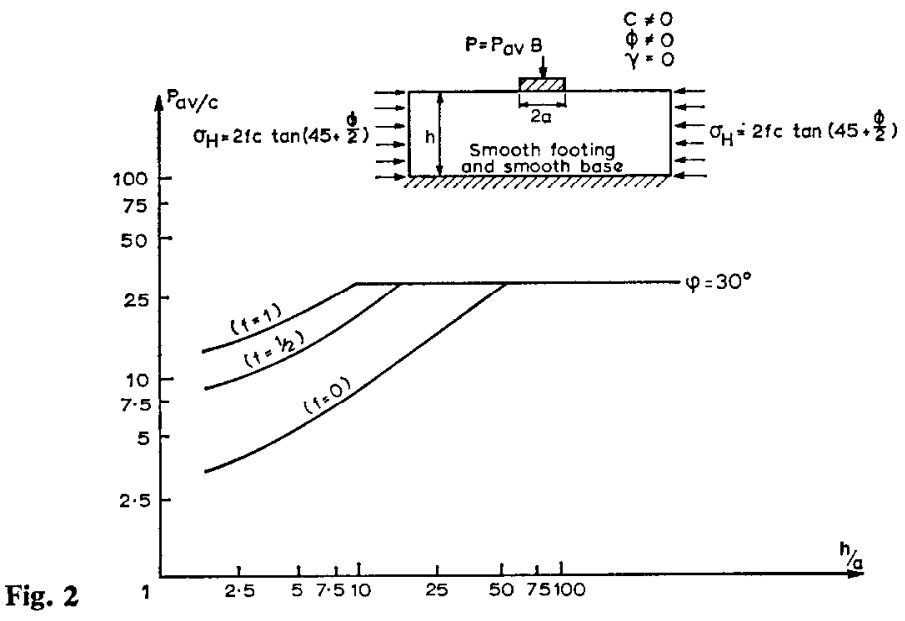

the average vertical stress on the footing which corresponds to an assumed average horizontal stress of $\sigma_{\mathrm{H}}^{\prime}$, with similar definitions for $P^{\prime \prime}{ }_{\mathrm{av}}$ and $\sigma^{\prime \prime}{ }_{\mathrm{H}}$. Then it may be shown that

$$
\frac{P_{a v}^{\prime}+c \cot \phi}{\sigma_{\mathrm{H}}^{\prime}+c \cot \phi}=\frac{P_{\mathrm{av}}^{\prime \prime}+c \cot \phi}{\sigma_{\mathrm{H}}^{\prime \prime}+c \cot \phi}
$$

Equation (1) can be used to relate Mandel and Salençon's numerical solution with the Writer's analytic solution. A comparison is shown in Fig. 1. Equation (1) can be also used to calculate the failure loads corresponding to an average horizontal stress which lies between the two extremes considered by Mandel and Salençon

$$
\sigma_{\mathrm{H}}=2 c \tan \left(\frac{\pi}{4}+\frac{\phi}{2}\right)
$$

and the Writer $\sigma_{\mathrm{H}}=0$. This has been done in Fig. 2 for the particular case of $\phi=30^{\circ}$.

\section{REFERENCES}

Booker, J. R. (1972). A method of integration of the equations of plasticity for a weightless cohesive frictional material. Q.Jnl Mech. Appl. Math. 25, Part 1.

\section{Apparatus for measuring volume change suitable for automatic logging}

ROWLANDS, G. O. (1972). Géotechnique 22, No. 3, 525-526.

\section{P. Darley, Earthworks and Foundations Division, Transport and Road Research Laboratory}

The Author has described a simple method for automatically recording volume changes in the triaxial test based on the Bishop self-compensating mercury constant-pressure unit and using a linear displacement transducer.

The Writer has developed a similar device shown in Fig. 1. Unlike the device described by Rowlands the apparatus has the advantage in that it uses a single spring which gives twice the sensitivity. Pot $B$ is fixed while pot $A$ is suspended from the spring by the core of the linear displacement transducer. The use of hook joints in the suspension facilitates the axial alignment of the core within the transducer body and reduces the effect on the core of lateral movements of the pot. 